\title{
An investigation into a suitable scintillator for localising neutron capture within a detector
}

\author{
Matthew J. I. Balmer ${ }^{\mathrm{a}, *}$, Kelum A. A. Gamage ${ }^{\mathrm{a}}$, Graeme C. Taylor ${ }^{\mathrm{b}}$ \\ ${ }^{a}$ Department of Engineering, Lancaster University, LA1 4YR, UK \\ ${ }^{b}$ Neutron Metrology Group, National Physical Laboratory, Teddington, TW11 OLW, UK
}

\begin{abstract}
Using Monte-Carlo modelling, an investigation into a suitable loaded scintillator for localising neutron capture in a novel neutron survey meter has been undertaken. A comparison of estimated neutron capture location in a scintillator with Geant 4 and MCNP simulations was undertaken and a good general agreement between the two models was observed. The interactions of $\gamma$ emissions from neutron capture in the scintillator are investigated. The results show that the $\gamma$ emission from neutron capture will not aid neutron capture localisation and will only inhibit it. It is observed that the lithium-loaded scintillator has the lowest neutron capture efficiency when compared with boron and gadolinium scintillators. However, it is the most promising of the detectors investigated in this research for use in a novel neutron survey meter design.
\end{abstract}

Keywords: Neutron detectors (cold, thermal, fast neutrons); Instrumentation for neutron sources; Scintillators, scintillation and light emission processes (solid, gas and liquid scintillators); Simulation methods and programs; Models and simulations.

\section{Introduction}

Previous work has highlighted that by taking into account the direction of incidence of a neutron field, a better estimate of the health risk to an individual exposed to the dose can be achieved [1]. A number of instruments have been previously developed to measure the directional component of a neutron field [2]. However, these instruments do not lend themselves well to portable, real-time applications. It has been identified that a theoretical design proposed by Taylor [3] could be realised into a portable, real-time instrument. The instrument proposed consists of a boron-loaded spherical scintillator of $20.32 \mathrm{~cm}$ diameter interrogated by multiple photomultiplier tubes (PMTs). A neutron entering the scintillator will undergo many elastic collisions, before finally coming to rest and being captured by boron.

By recording a large number of neutron capture events within the scintillator, a pattern related to energy and direction of incidence of the neutron field to the detector can be observed. An artificial neural network was trained with data obtained through Monte-Carlo simulations and in this proof-of-concept it was shown that an estimate of effective dose could be achieved using this instrument.

However, this proof-of-concept has not yet been realised into a physical instrument. To allow the instrument to be realised into a working detector, a novel technique will need to be identified to localise the neutron capture within the scintillator.

A high photon yield from the scintillator is highly desirable to obtain a good signal to noise ratio of photons from scintillation as a result of neutron capture. The strong quenching in a boron-loaded scintillator greatly reduces the photon yield [4], to a light intensity of $60 \mathrm{keVee}$ (electron equivalent). It is anticipated that the interior of the detector would be non-reflective and so considering a typical PMT of around $1-2 \mathrm{~cm}$ radius, only a small portion of the scintillation light will be collected by the light detectors around the perimeter of the detector.

*E-mail address: m.balmer@lancaster.ac.uk (M. J. I. Balmer) 


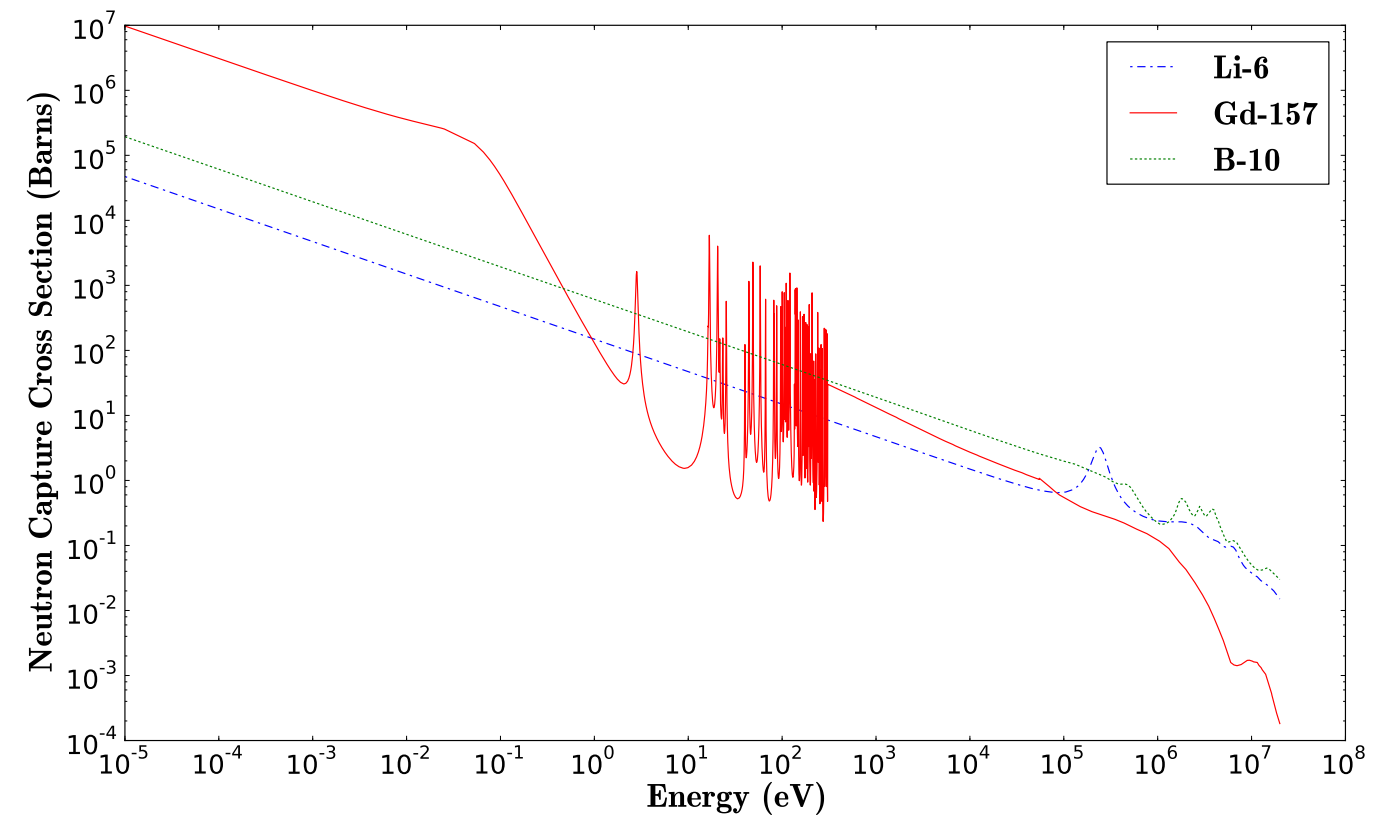

Figure 1: Neutron capture cross section for lithium, boron and gadolinum using data from ENDF/B-VII.r1.

In recent developments in the field of crystal scintillators, $\mathrm{Cs}_{2} \mathrm{LiYCl}_{6}: \mathrm{Ce}$ (CLYC) is perhaps the most promising [5]. CLYC has been shown to have high photon yield, good light transmittance and a lithium loading for neutron capture. However, growing these crystals to the desired sizes is not currently possible [6] [7].

Plastic scintillators are advantageous in many ways, boasting fast response times, significantly less hazardous materials, low cost of fabrication, and high light yields [8]. However scaling a plastic detector to a large size has associated problems, a heavier mass compared to that of a liquid scintillator and poor light transmittance due to the loading element [8]. A number of smaller crystal or plastic scintillators could be configured into an array to create a larger detector capable of estimating dose at high energies. However, such an instrument would as well as being significantly more complex, probably prove cost-prohibitive.

A liquid scintillator with a suitable loading for neutron capture shows the most promise for use in this design. The three main choices of loading for neutron capture within a liquid scintillator are boron, lithium and gadolinium due to their high cross section allowing for efficient neutron capture. This paper will investigate the suitability of a liquid scintillator with each of these loading elements in, with a view to localising neutron capture within the scintillator.

\section{Liquid scintillators for neutron capture}

\subsection{Boron-loaded scintillator}

Boron-loaded scintillators are typically loaded with upto $5 \%$ boron. The boron capture reaction is shown in equation 1 ( $6 \%$ branching ratio) and equation 2 (94\% branching ratio).

$$
\begin{gathered}
{ }^{1} n+{ }^{10} \mathrm{~B}->{ }^{7} \mathrm{Li}(0.84 \mathrm{MeV})+\alpha(1.47 \mathrm{MeV}) \\
{ }^{1} n+{ }^{10} \mathrm{~B}->{ }^{7} \mathrm{Li}+\alpha+\gamma(0.477 \mathrm{MeV})
\end{gathered}
$$

A boron-loaded scintillator presents a promising detector for use in this application with a reasonably high energy $\alpha$ emitted as a result of neutron capture. However as has previously been mentioned, the strong quenching within 
Table 1: Fractional mass composition of a typical boron-loaded liquid scintillator of density $0.92 \mathrm{~g} / \mathrm{cm}^{3}$.

\begin{tabular}{c|ccccc}
\hline Element & $\mathrm{C}$ & $\mathrm{H}$ & $\mathrm{O}$ & ${ }^{10} \mathrm{~B}$ & ${ }^{11} \mathrm{~B}$ \\
\hline Fractional mass & 0.624 & 0.09 & 0.236 & 0.045 & 0.005 \\
\hline
\end{tabular}

the scintillator due to the heavy lithium particle from the capture reaction, means that only $60 \mathrm{keVee}$ of light is emitted from neutron capture. For the commercially available BC-523A (with 5\% boron loading), this equates to approximately 670 photons being spread isotropically from the neutron capture location. More recent development of EJ309:B5 [9] has a higher photon yield of around $120 \mathrm{keVee}$ from neutron capture, equating to around 1380 photons.

Another consideration for a boron-loaded scintillator is the possibility of the $477 \mathrm{keV} \gamma$ Compton scattering within the scintillator and presenting a false signal to a capture localising algorithm. It has been noted that in smaller detectors of a $5 \mathrm{~cm}$ diameter, this is not a problem [10]. However, for the larger detector volumes being considered within this design, this is worthy of consideration.

\subsection{Gadolinium-loaded scintillator}

With a high cross section (as shown in Figure 1), gadolinium allows for efficient neutron capture detection. Within the scope of this work, the prompt emission of high energy $\gamma$ from neutron capture may cause problems with localising neutron capture. This $\gamma$ will travel away from the capture location before Compton scattering with an electron. The location of this Compton scattering could be a considerable distance from the location of the neutron capture. With the original proposed design, the capture was voxelised into $2 \times 2 \times 2 \mathrm{~cm}^{3}$ voxels. If a sufficient number of high energy Compton scatters occur less than $2 \mathrm{~cm}$ from the neutron capture location, this could make the scintillator worthy of consideration.

\subsection{Lithium-loaded scintillator}

The two previous liquid scintillators discussed, emit a $\gamma$ as a result of neutron capture. As shown in equation 3 , there is no $\gamma$ emission from the lithium neutron capture reaction.

$$
{ }^{1} n+{ }^{6} \mathrm{Li}->t(2.73 \mathrm{MeV})+\alpha(2.05 \mathrm{MeV})
$$

The lack of $\gamma$ from neutron capture makes ${ }^{6} \mathrm{Li}$ a promising candidate for use in this design. The lighter tritium and $\alpha$ particles from the capture reaction mean that there is less quenching of the scintillation from neutron capture. As a result of this it is estimated ten times as much light is emitted from neutron capture [11].

Of the three loading elements considered within this paper, it can be seen in Figure 1 lithium has a significantly lower cross section. The impact of this is that the previously observed limit of directional capture pattern observed with BC-523A could be much lower. Although no lithium-loaded scintillators are commercially available, previous research has shown that such a scintillator could be manufactured [12] [11].

\section{Detector modelling and simulation}

\subsection{Establishing a suitable model of the detector}

Monte-Carlo simulation codes allow experimental situations to be modelled and evaluated before experimental work is undertaken. These codes are used in a wide range of applications including, high energy physics, medicine, nuclear reactor design through to dosimetry estimations. Arguably one of the most commonly used Monte-Carlo codes for low energy neutron transportation simulation is MCNP [13]. This well established code is considered well bench marked for these low energy neutron simulations. However the current version of MCNP does not allow for transport of low energy photons (i.e. visible light). Within the scope of this work, modelling of scintillation and low energy photons is highly desirable. Geant4 [14] [15] can be used to simulate both transport of neutrons and photons in a scintillator. The Geant 4 toolkit uses the object-orientated programming language $\mathrm{C}++$ and allows the user to write fully customised code.

By using the photon transport models within Geant 4 , it has previously been possible to model liquid scintillators and to investigate photon production and pulse shapes [16] [17]. However the detectors that were modelled were 


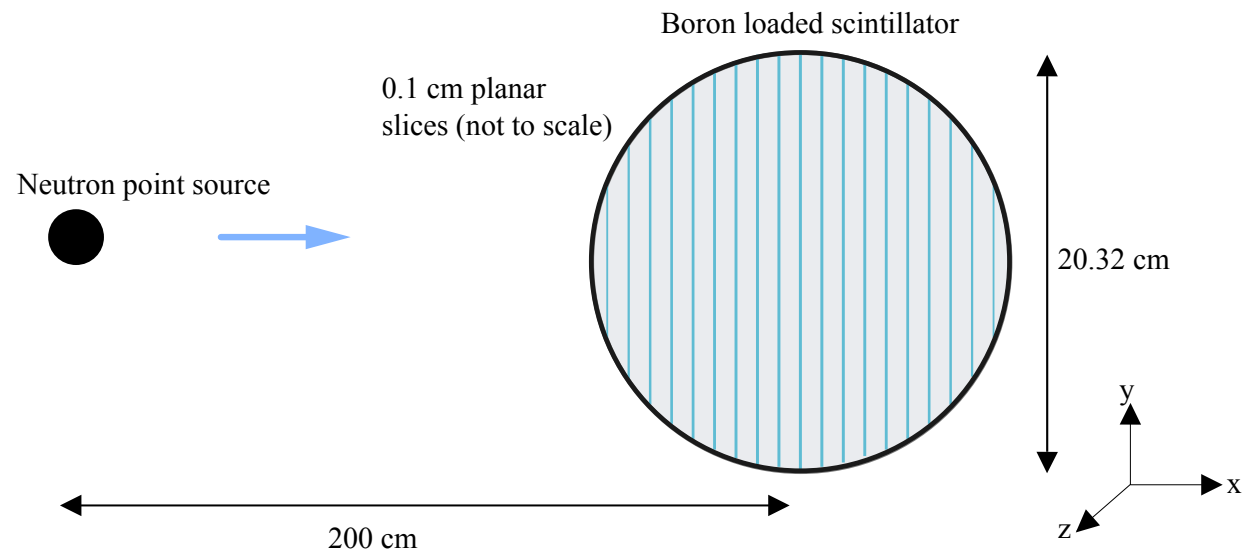

Figure 2: Detector geometry under simulation with neutrons emitted in the direction of the $\mathrm{x}$-axis.

concerned with proton recoil energy and did not use loaded scintillators for neutron capture. At lower neutron energies for thermal neutron simulations, there are conflicting results when compared to simulations performed with MCNP [18]. However, no further results have been published since Geant4 v4.9.2. The latest stable release of Geant4 is at v4.9.6.p02. It was felt that for this work, before proceeding with simulations written using Geant4, some comparison should be undertaken between Geant 4 and MCNP, focusing on thermal neutrons. By considering the location of neutron capture within the scintillator, this would provide a good comparison of both the higher energy physics models for proton recoil, as well as the lower energy thermal transport of the neutrons.

All simulations under consideration in this work were run on a $3.2 \mathrm{GHz}$ processor running the Linux based operating system, Ubuntu 12.04. The codes used were Geant4 version 4.9.6.p02 and MCNP5 v1.60.

The geometry of the detector simulated can be seen in Figure 2. A typical boron-loaded scintillator with a density of $0.92 \mathrm{~g} / \mathrm{cm}^{3}$ and a diameter of $20.32 \mathrm{~cm}$ was placed inside a vacuum. The material fractional masses listed in Table 1 were chosen to be that of a typical boron-loaded scintillator used for neutron capture such as BC-523A. The centre of the spherical scintillator was at coordinate $(0,0,0) \mathrm{cm}$. A mono-energetic, mono directional source was located at $(-200,0,0) \mathrm{cm}$, emitting neutrons in the direction of the $\mathrm{x}$-axis.

Geant 4 allows for full customisation of the simulation through a series of compulsary and non-compulsary C++ classes. A custom physics list was created for the simulations. The high-precision neutron physics models were chosen for the simulation of neutrons from thermal to an upper energy range of $20 \mathrm{MeV}$. The following Geant 4 physics was included; G4NeutronHPElastic, G4NeutronHPInelastic, G4NeutronCaptureAtRest and G4NeutronHPCapture. The neutron cross section data in the Geant4 G4NDL4.2 library, is largely based on ENDF/B-VII.r1.

G4NeutronThermalScattering was used with a thermal treatment of hydrogen using the G4Element TS_H_of_Polyethylene. A mininum energy of $4 \mathrm{eV}$ was set for the elastic scattering and maximum energy of $4 \mathrm{eV}$ for thermal scattering.

In MCNP materials were simulated using the ENDF/B-VII.0 neutron cross section tables at temperature 293.13 $\mathrm{K}$. To handle low energy thermal scattering of neutrons below $5 \mathrm{eV}$, MCNP has thermal treatment for hydrogen in polyethylene. For $\mathrm{s}(\alpha, \beta)$ thermal treament, poly.01t was included in the MCNP input file. It was observed that all simulations using MCNP had a statistical uncertainty of less than $0.01 \%$. Using the particle tracking file (PTRAC), neutron capture events were counted, recording the $(\mathrm{x}, \mathrm{y}, \mathrm{z})$ location in $\mathrm{cm}$ of the neutron capture within the detector. It is noted that Geant 4 and MCNP interpolate the ENDF neutron cross section data with a different number of data points.

\subsection{A comparison of neutron capture location using Geant and MCNP}

Identical geometries and material specifications were modelled to allow comparison of the two codes. Both simulation codes were configured to record the three dimensional neutron capture location within the scintillator. To minimise statistical variation, every simulation recorded at least $10^{6}$ neutron capture events. Planar slices $0.1 \mathrm{~cm}$ thick along the $\mathrm{x}$-axis of the detector were created and neutron capture within these slices was counted. 


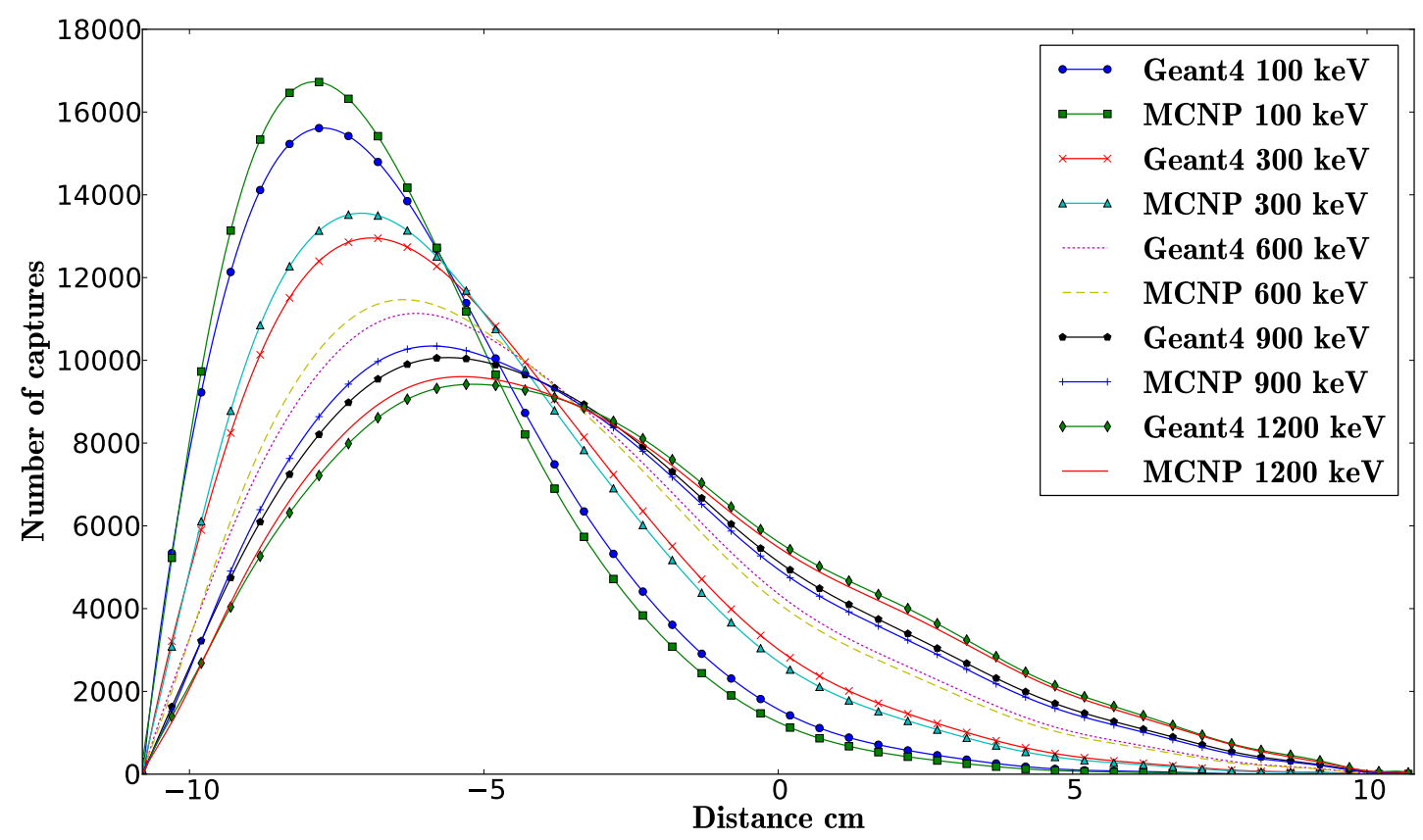

Figure 3: A comparison between MCNP and Geant4 of neutron captures recorded in $0.1 \mathrm{~cm}$ slices for $100 \mathrm{keV}, 300 \mathrm{keV}, 600 \mathrm{keV}, 900 \mathrm{keV}$ and $1200 \mathrm{keV}$ neutron sources.

Table 2: The table shows testing correlation of the polynomial fitting function to the data. The $\mathrm{r}^{2}$ value is provided to show the closeness of fit of Geant 4 and MCNP at each neutron source energy simulated.

\begin{tabular}{clcc}
\hline Energy keV & \multicolumn{2}{l}{$\begin{array}{l}\text { Pearson correlation coefficient between fit and data } \\
\text { Geant4 }\end{array}$} & $\mathrm{r}^{2}$ value \\
\hline 100 & 0.9909 & 0.9911 & 0.9929 \\
300 & 0.9922 & 0.9919 & 0.9954 \\
600 & 0.9914 & 0.9913 & 0.9964 \\
900 & 0.9904 & 0.9904 & 0.9966 \\
1200 & 0.9897 & 0.9894 & 0.9972 \\
\hline
\end{tabular}

Figure 3 shows the neutron capture location along the x-axis, for $100 \mathrm{keV}, 300 \mathrm{keV}, 600 \mathrm{keV}, 900 \mathrm{keV}$ and 1200 $\mathrm{keV}$ neutron sources, using MCNP and Geant4. A $17^{\text {th }}$ order polynomial fitting function was used for the data sets. An example of the spread of the data can be seen in Figure 4. Due to the non linear nature of the data set a Pearson correlation coefficient test was used to ensure that the fitting function had a suitable fit with each data set. The outcome of a Pearson correlation coefficient test is a coefficient between 0 and 1 , where a number approaching 1 signifies a strong correlation between the two variables and 0 signifies no correlation. It can be seen from Table 2 that for each of the neutron energies a good polynomial fit has been achieved.

For numerical analysis of the fit between Geant4 and MCNP, at each energy, a linear regression test was performed for each neutron source energy. Figure 5 shows an example of the linear regression test undertaken for each neutron source energy. The number of neutrons captured in each slice was plotted on the x-axis for MCNP and the y-axis for Geant4. If the two models were to output exactly the same data, the graph would perfectly fit the line of $y=x$. Linear regression was tested between the two variables (MCNP and Geant4 data) and the expected value of $y=x$. The $r^{2}$ value for each neutron source energy can be seen in Table 2.

From Table 2 and Figure 3 it can be seen that Geant 4 and MCNP are in general agreement in terms of neutron 


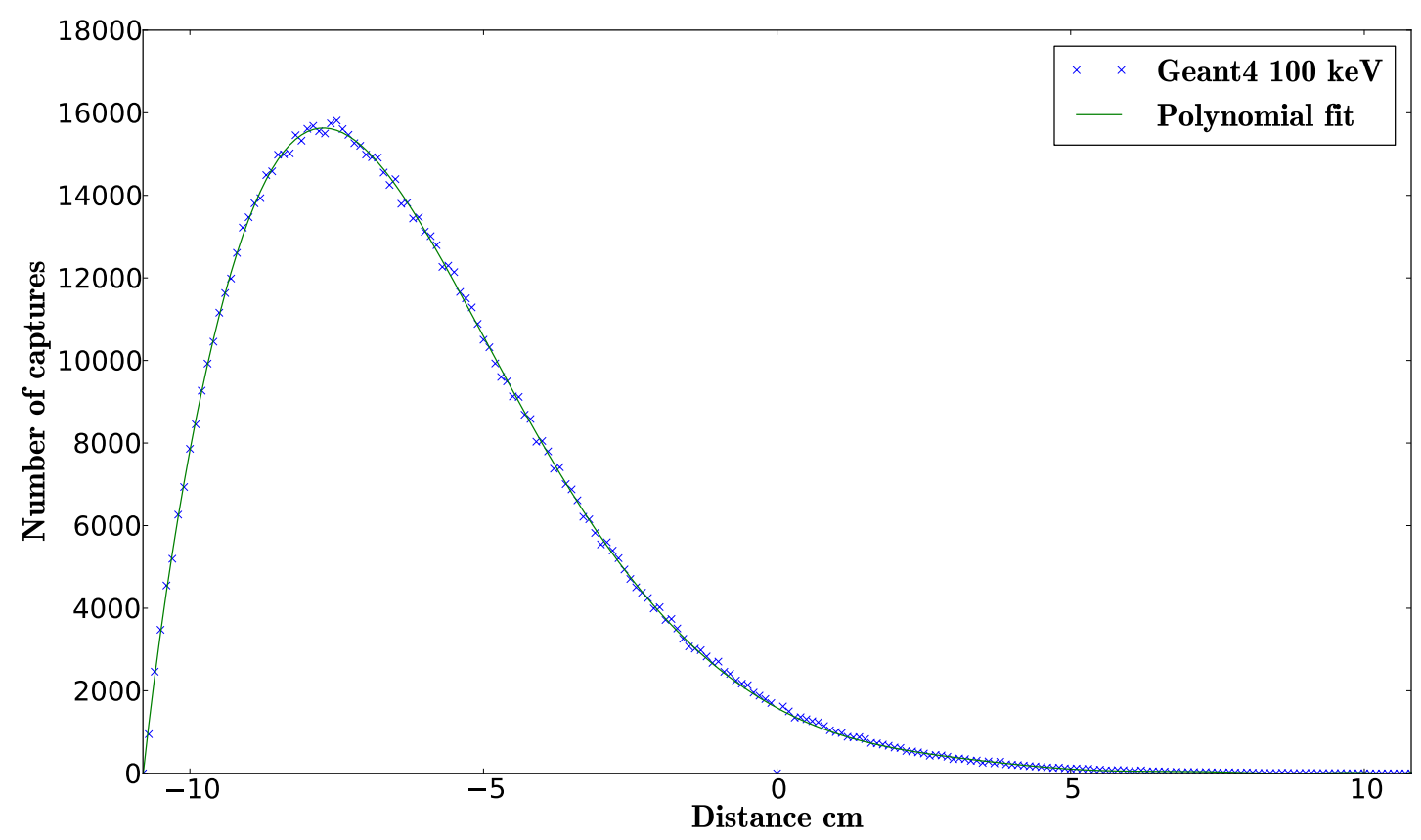

Figure 4: Neutron captures recorded in $0.1 \mathrm{~cm}$ slices for $100 \mathrm{keV}$. The data was fitted using a polynomial fitting function.

capture with respect to depth in the detector. From the $r^{2}$ values in Table 2 it can be seen that at lower energies there is less agreement between the two models. It can be seen in Figure 3 that the location along x-axis of the neutron capture are in agreement, however it can be seen that Geant 4 estimates fewer neutron captures at this peak capture location. Geant4 estimates that more neutrons will be captured further along the $\mathrm{x}$-axis into the scintillator.

\section{Investigating a suitable liquid scintillator}

In order to assess the suitability of different loading materials in a scintillator for this application, a number of factors must be taken into consideration. Perhaps the most important factor is the investigation of $\gamma$-ray emission from capture. It has previously been noted that for boron-loaded scintillators investigating neutron capture, with a detector of size under consideration in this study, there is a $78 \%$ chance of a $\gamma$ interaction in the scintillator resulting in greater than $50 \mathrm{keV}$ being deposited [10]. Within the scope of this work, it is necessary to not only understand the probability of a $\gamma$ interaction, but also to understand the likelihood of where this event will occur, and the likely energy that will be deposited.

The impact of a Compton scatter of a $\gamma$ within a detector impeding the ability to localise neutron capture is not currently fully understood. However within the scope of this work it has been decided to investigate which scintillator exhibits the lowest frequency of $\gamma$ interaction within the scintillator as a function of distance from the neutron capture location.

Capture efficiency of the detector is also of importance, particularly in a large size detector. A low capture efficiency may impede the instrument's ability to detect the energy and/or direction of the neutron field under investigation. It can be seen in Figure 1 that the neutron capture cross section of lithium is significantly lower than the two other loading elements considered in this research. It was felt neccesary to investigate the impact of this on the neutron capture efficiency of the scintillator. 


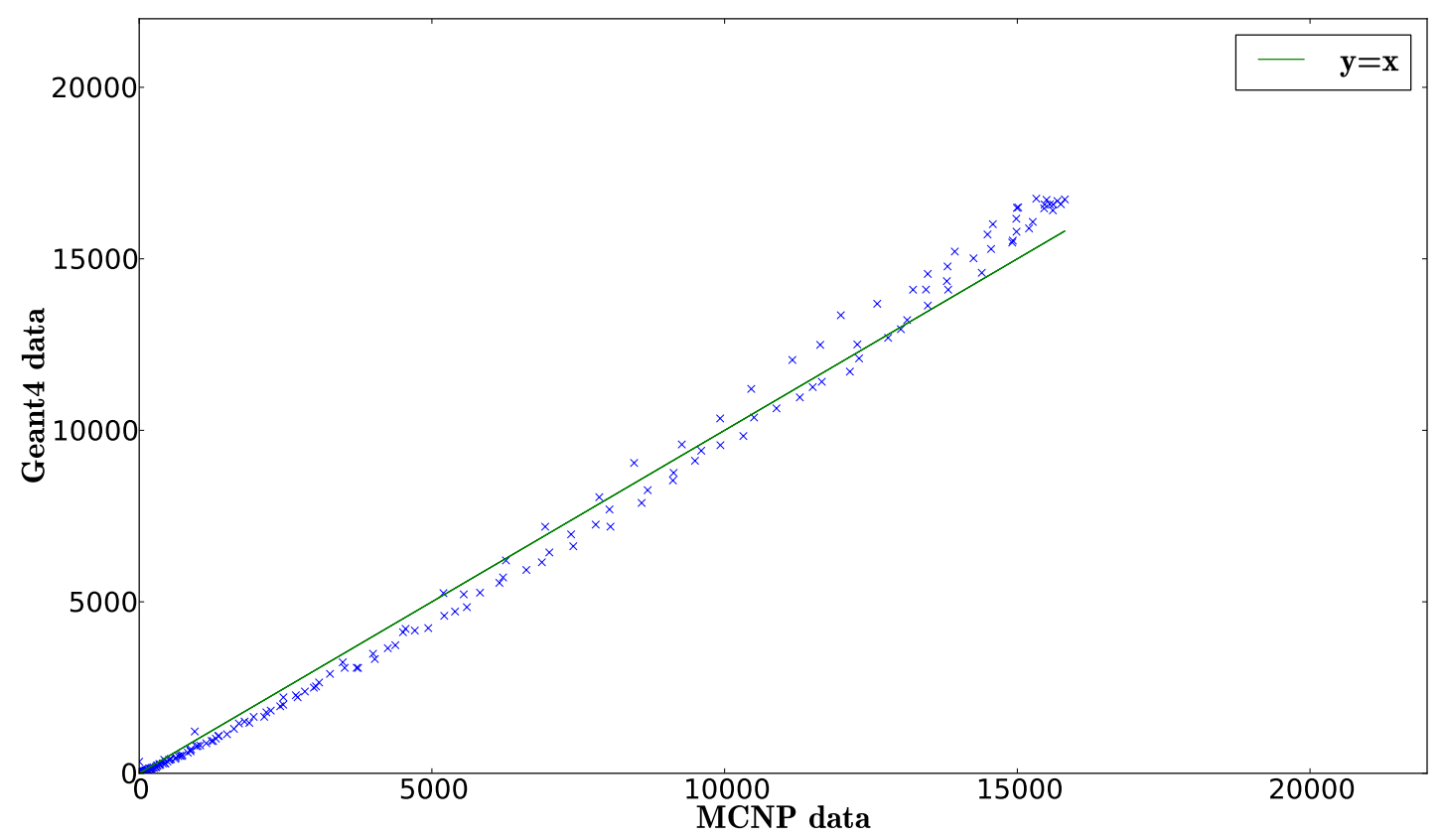

Figure 5: A comparison of neutron captures in $0.1 \mathrm{~cm}$ slices through the detector models in Geant 4 and MCNP for a $100 \mathrm{keV}$ source. The line $\mathrm{y}=\mathrm{x}$ is set as the ideal, whereby both models respond with the same number of neutron captures. The deviation from this ideal response was testing using linear regression techniques.

Table 3: Fractional mass composition of lithium and gadolinium scintillators simulated in this work.

\begin{tabular}{ccc}
\hline Element & Fractional mass \% for Gd loading & Fractional mass \% for ${ }^{6} \mathrm{Li}$ loading \\
\hline $\mathrm{C}$ & 0.8525 & 0.858 \\
$\mathrm{H}$ & 0.1228 & 0.105 \\
$\mathrm{O}$ & 0.0144 & 0.0304 \\
$\mathrm{~N}$ & 0.0003 & 0.0026 \\
Loading & 0.01 & 0.004 \\
\hline
\end{tabular}

\subsection{Simulation parameters}

The same model detailed in Figure 2 was used for the simulations undertaken in this section. The boron-loaded scintillator used was the same as that shown in Table 1. The gadolinium and lithium-loaded scintillators were based on cocktails described in [12] [19], these are shown in Table 3. These scintillator compositions were chosen as ones which have proven ability and could be suited for use in this work. Each of the scintillators were given a density of $0.92 \mathrm{~g} / \mathrm{cm}^{3}$. Differences in neutron capture efficiency have previously been noted between MCNP and Geant4 simulations [18]. It was decided, for neutron capture efficiency simulations, to use both codes to ensure that the general trend was the same between the two codes.

\subsection{Neutron capture efficiency}

To investigate the capture efficiency of the detector, a neutron was emitted from a point source at $-200,0,0$ towards the detector in a single event, where $2 \times 10^{6}$ events were simulated for each detector. Neutron energies of $100 \mathrm{keV}, 1$ $\mathrm{MeV}$ and $10 \mathrm{MeV}$ were simulated for the three different scintillators. The number of neutrons captured were recorded using Geant4 and MCNP. The neutron capture efficiency was defined as number of captures, divided by the total 
Table 4: Neutron capture efficiency for neutron energies of $100 \mathrm{keV}, 1 \mathrm{MeV}$ and $10 \mathrm{MeV}$. All values given are percentages.

\begin{tabular}{ccccc}
\hline Loading & Simulation Code & $100 \mathrm{keV}$ & $1 \mathrm{MeV}$ & $10 \mathrm{MeV}$ \\
\hline Lithium & MCNP & 40.4 & 49.7 & 18.1 \\
& Geant4 & 34.4 & 38.2 & 21.2 \\
\hline Boron & MCNP & 63.4 & 52.7 & 17.0 \\
& Geant4 & 56.7 & 48.5 & 20.3 \\
\hline Gadolinium & MCNP & 50.9 & 55.9 & 23.7 \\
& Geant4 & 53.5 & 57.6 & 29.8 \\
\hline
\end{tabular}

number of neutrons simulated (including neutrons which escaped without capture). The statistical uncertainties were observed to be less than $0.001 \%$ for these simulations.

\subsection{Gamma interaction in scintillator}

The Geant4 General Particle Source was used to generate the source particle a $\gamma$, with isotropic emission for investigation of $\gamma$ interactions in the scintillator. For each scintillator loading, a different $\gamma$ energy was simulated. In $94 \%$ of neutron capture events by boron, a $\gamma$ is emitted, making this the most important $\gamma$ energy to consider for this scintillator.

In a lithium-loaded scintillator, although a significantly lower cross section (0.33 Barns), hydrogen capture will lead to emission of a $2.2 \mathrm{MeV} \gamma$. The resultant $2.2 \mathrm{MeV} \gamma$ from this hydrogen neutron capture was used for the lithium simulations. With gadolinium a number of prompt $\gamma$ emissions occur as a result of neutron capture, $1 \mathrm{MeV}, 2 \mathrm{MeV}$ and 7.5 MeV $\gamma$ energies were chosen for simulation this scintillator loading.

The location of the particle source was chosen at $(0,0,-5)$ and $2 \times 10^{6}$ events were simulated for each detector. Previous work [2] has shown that for neutron energies from $10 \mathrm{keV}$ to $1 \mathrm{MeV}$ this front region of the detector is the most likely location for neutron capture to take place. The number of $\gamma$ escaping the detector without any interaction within the detector was recorded. When a $\gamma$ did Compton scatter in the detector, the resulting electron energy was recorded as well as the distance from the origin of the $\gamma$.

\section{Results}

The capture efficiency of the different scintillators can be seen in Table 4. It can be seen that the higher loading by mass of boron equates to a similar loading capture efficiency of the lower percentage loading of gadolinium. As would be expected, with the lowest cross section of the three loading elements, lithium, has the lowest capture efficiency. As has previously been observed [18] there is a difference of around $5 \%$ between MCNP and Geant 4 in terms of neutron capture efficiency. Although the absolute efficiencies are observed to be different between the two codes, it can be seen that there is a good agreement between the two codes in terms of the trend for neutron capture efficiency against energy, for each scintillator simulated. The reason for these differences is thought to be in the way the two codes handle weight cut off of neutrons.

Within the boron-loaded scintillator, it can be seen in Table 5 that $42 \%$ of the $478 \mathrm{keV} \gamma$ emitted from neutron capture by boron escape without interactions. Figure 6 and 7 show there is a high probability of this particle interacting and depositing a significant amount of energy greater than $2 \mathrm{~cm}$ away from the neutron capture location. As a result of these $\gamma$ interactions in the scintillator, multiple scintillations will occur in the scintillator. It may not be possible to localise neutron capture from multiple scintillation events.

For a gadolinium-loaded scintillator, it can be seen that as the $\gamma$ emission energy increases the probability of the $\gamma$ interacting in the detector reduces. However, it can be seen in Figure 6 and 7 that there is a high probability of this $\gamma$ interacting more than $2 \mathrm{~cm}$ from the neutron capture location. It can be seen that it would be impossible to localise neutron capture in a gadolinium scintillator from the $\gamma$ Compton scattering in the scintillator.

In the lithium-loaded scintillator hydrogen capture is a low probability. It can be seen that the unwanted $2.2 \mathrm{MeV}$ $\gamma$ from this capture reaction escapes the scintillator $66 \%$ of the time without interaction. This further reduces the impact of a low probability neutron capture by hydrogen. 
Table 5: Percentage of $\gamma$ particles escaping the detector without under going a Compton scattering event.

\begin{tabular}{ccl}
\hline Scintillator loading & $\gamma$ energy used for simulation & $\begin{array}{l}\text { Percentage of } \gamma \text { escaping } \\
\text { without interaction }\end{array}$ \\
\hline Boron & $478 \mathrm{keV}$ & $42 \pm 0.11$ \\
Gadolinium & $1 \mathrm{MeV}$ & $52 \pm 0.10$ \\
Gadolinium & $2 \mathrm{MeV}$ & $64 \pm 0.09$ \\
Gadolinium & $7.5 \mathrm{MeV}$ & $80 \pm 0.08$ \\
Lithium & $2.2 \mathrm{MeV}$ & $66 \pm 0.9$ \\
\hline
\end{tabular}

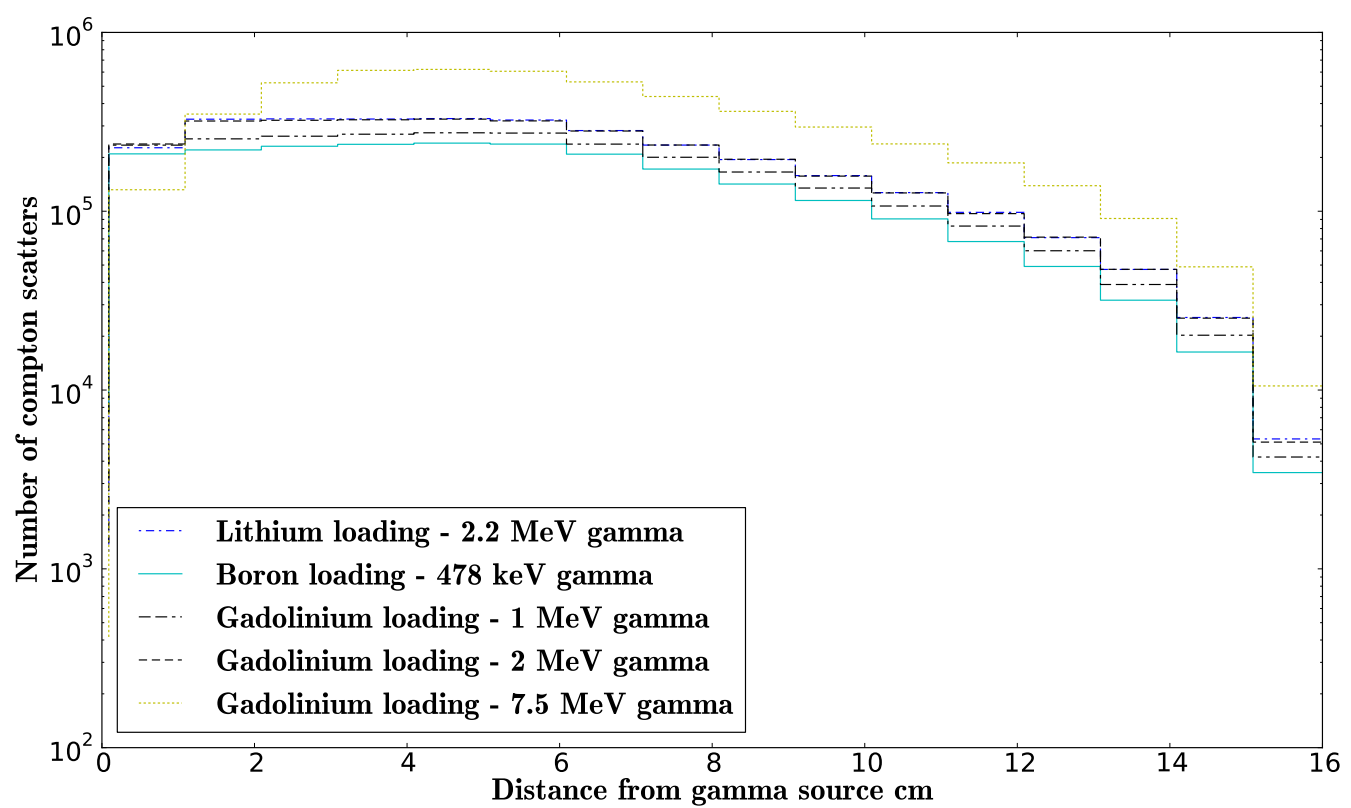

Figure 6: Investigating distance of Compton scatter from the $\gamma$ source within a liquid scintillator with three different loadings; boron, lithium and gadolinium.

\section{Conclusion}

In this work, three different loading elements have been considered for use in a scintillator suitable for localising neutron capture. Before any simulations were undertaken to investigate the suitability of these detectors, a comparison of Geant4 was undertaken against MCNP to validate the detector model written within Geant4. It was shown that the two models were in general agreement with each other, when considering the neutron capture against depth in the scintillator. In future work when establishing a method of localising neutron capture in the scintillator, comparisons of predicted neutron capture location against simulated data will be crucial. Comparison will be able to now be undertaken with confidence in the simulated data due to the general agreement of the two models.

Although gadolinium has a high cross section for neutron capture, the prompt $\gamma$ emission from neutron capture has been shown to Compton scatter too far away from the neutron capture location to allow the capture to be localised. With a boron-loaded scintillator the two major disadvantages are the $\gamma$ emission from $94 \%$ of captures, of which 42 $\%$ of these will Compton scatter within the detector. In such cases, the impact would either be false location of the neutron capture, or if a suitable pulse height gating algorithm could be employed, a reduced efficiency of the detector. Achieving good pulse shape discrimination on such small pulses of light could prove difficult which would also reduce the detector efficiency.

The most promising of the three scintillators investigated in this work is the ${ }^{6} \mathrm{Li}$-loaded liquid scintillator. The lack 


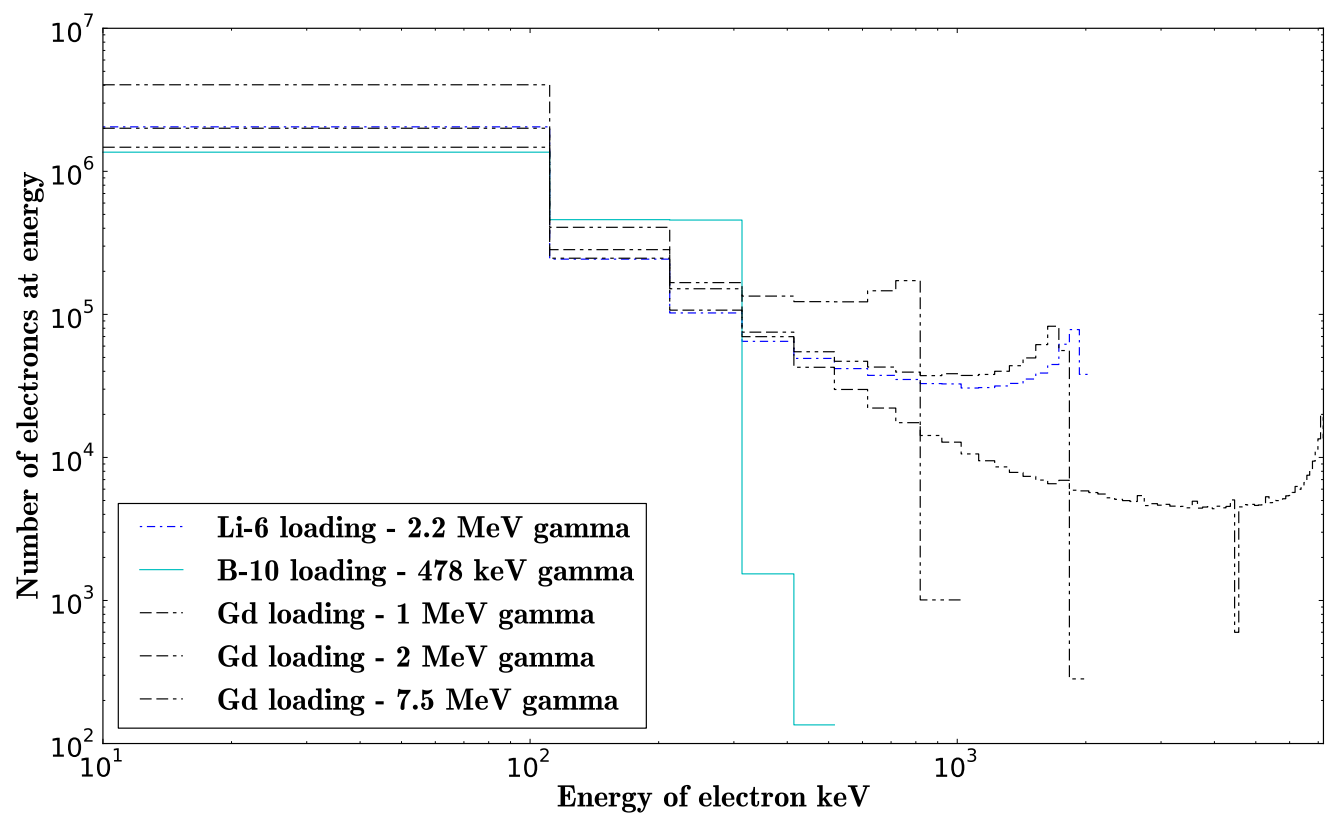

Figure 7: Energy of electron Compton scattered by $\gamma$ within a liquid scintillator with three different loadings; boron, lithium and gadolinium.

of $\gamma$ from a neutron capture reaction and high energy, light, particles emitted from this reaction make it a promising choice for this work. It has also been shown that although lithium has a lower cross section, compared with boron, it still has satisfactory neutron capture efficiency. However, it is worth noting that currently no commercially available ${ }^{6}$ Li-loaded liquid scintillator exists. Developing a suitable scintillator and characterising such a scintillator would need to take place to establish its suitability.

\section{Acknowledgments}

The authors would like to acknowledge the funding support from EPSRC and National Physical Laboratory, Teddington, UK. We also acknowledge the help and advise of Prof. Malcolm Joyce at Lancaster University and Dr. Nigel Hawkes at the National Physical Laboratory. The authors acknowledge the use of the package Matplotlib for all plots in this research [20].

\section{References}

[1] D.T. Bartlett et al. The importance of the direction distribution of neutron fluence, and methods of determination. Nuclear Instruments and Methods in Physics Research Section A: Accelerators, Spectrometers, Detectors and Associated Equipment, 476(1-2):386 - 394, 2002.

[2] M.J.I. Balmer et al. Critical review of directional neutron survey meters. Nuclear Instruments and Methods in Physics Research Section A: Accelerators, Spectrometers, Detectors and Associated Equipment, 735:7-11, January 2014.

[3] G.C. Taylor. Design of a direction-dependent neutron dosimeter. Radiation Measurements, 45(10):1301-1304, December 2010.

[4] L. Swiderski et al. Boron-10 loaded BC523A liquid scintillator for neutron detection in the border monitoring. IEEE Transactions on Nuclear Science, 55(6):3710-3716, December 2008.

[5] J. Glodo et al. Development of Cs2LiYCl6 scintillator. Journal of Crystal Growth, March 2013.

[6] C.W.E van Eijk. Inorganic scintillators for thermal neutron detection. IEEE Transactions on Nuclear Science, 59(5):2242 - 2247, October 2012.

[7] B. Ayaz-Maierhafer et al. Transmission and signal loss in mask designs for a dual neutron and gamma imager applied to mobile standoff detection. Nuclear Instruments and Methods in Physics Research Section A: Accelerators, Spectrometers, Detectors and Associated Equipment, 712:1-8, June 2013.

[8] R.D. Breukers et al. Transparent lithium loaded plastic scintillators for thermal neutron detection. Nuclear Instruments and Methods in Physics Research Section A: Accelerators, Spectrometers, Detectors and Associated Equipment, 701:58-61, February 2013. 
[9] L. Swiderski et al. Further study of boron-10 loaded liquid scintillators for detection of fast and thermal neutrons. IEEE Transactions on Nuclear Science, 57(1):375-380, February 2010.

[10] S.C. Wang et al. A feasibility study of boron-loaded liquid scintillator for the detection of electron anti-neutrinos. Nuclear Instruments and Methods in Physics Research Section A: Accelerators, Spectrometers, Detectors and Associated Equipment, 432(1):111-121, 1999.

[11] B.M. Fisher et al. Fast neutron detection with 6Li-loaded liquid scintillator. Nuclear Instruments and Methods in Physics Research Section A: Accelerators, Spectrometers, Detectors and Associated Equipment, 646(1):126-134, August 2011.

[12] C.D. Bass et al. Characterization of a 6Li-loaded liquid organic scintillator for fast neutron spectrometry and thermal neutron detection. Applied Radiation and Isotopes, 77:130-138, July 2013.

[13] F. Brown et al. A general monte carlo n-particle transport code, version 5. Los Alamos LA-UR-03-1987.

[14] J. Allison et al. Geant4 developments and applications. IEEE Transactions on Nuclear Science, 53(1):270-278, February 2006.

[15] S. Agostinelli et al. Geant4 - a simulation toolkit. Nuclear Instruments and Methods in Physics Research Section A: Accelerators, Spectrometers, Detectors and Associated Equipment, 506(3):250-303, July 2003.

[16] S.F. Naeem et al. Validation of Geant4 and MCNPX-PoliMi simulations of fast neutron detection with the EJ-309 liquid scintillator. Nuclear Instruments and Methods in Physics Research Section A: Accelerators, Spectrometers, Detectors and Associated Equipment, 714:98-104, June 2013.

[17] N. Patronis. Aspects of GEANT4 monte-carlo calculations of the BC501A neutron detector. Nuclear Instruments and Methods in Physics Research Section A: Accelerators, Spectrometers, Detectors and Associated Equipment, 578(1):351-355, July 2007.

[18] C. Guardiola et al. Geant4 and MCNPX simulations of thermal neutron detection with planar silicon detectors. Journal of Instrumentation, 6(09):T09001-T09001, September 2011.

[19] H.Y. Xing et al. Simulation of large-scale fast neutron liquid scintillation detector. Chinese Physics C, 37(2):026003, February 2013.

[20] J. D. Hunter. Matplotlib: A 2d graphics environment. Computing In Science E Engineering, 9(3):90-95, 2007. 